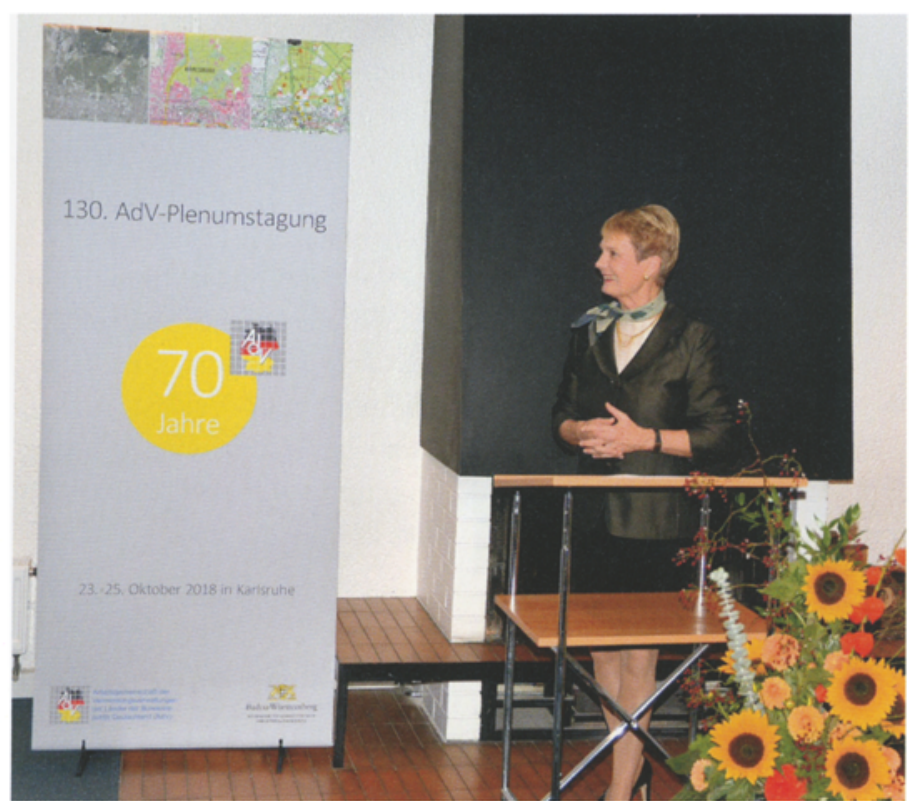

Abb. 2: Staatssekretärin Friedlinde Gurr-Hirsch anlässlich der Festansprache zum 70-jährigen Bestehen der AdV

technische Entwicklungen enorm. Die durch das amtliche deutsche Vermessungswesen zu erfüllenden Aufgaben unterliegen einem Wandel, dessen sich die AdV in der Tagung in Karlsruhe annimmt. Auch in Zukunft wird

\section{Politisches Programm des DDGI veröffentlicht}

Der DDGI - Deutscher Dachverband für Geoinformation e. V. verfolgt das Ziel, die generelle Bedeutung von Geoinformationen herauszustellen, ihre Nutzung zu verstärken und die Rahmenbedingungen für die öffentlichen Geoinformationen in Deutschland $\mathrm{zu}$ verbessern. Er versteht sich als „Meetingpoint“ und Technologienetzwerk für Forschung und Entwicklung, für Lehre, behördliche Institutionen, den Handel, die Wirtschaft und Privatpersonen aus der Geoinformatikbranche. Der Dachverband begleitet die Arbeit des interministeriellen Ausschusses für Geoinformationswesen IMAGI und beteiligt sich aktiv in der Kommission für Geoinformationswirtschaft.

Mitglieder, Beirat und Vorstand des Dachverbandes haben nach Auswertung des Koalitionsvertrages das neue politische Programm des DDGI erarbeitet. Es
- Harmonisierung und Erweiterung der nationalen und europäischen Informationsinfrastrukturen

Das breite bereits vorhandene Spektrum von Infrastrukturen soll genutzt, ausgebaut und verfestigt werden.

- Kompetenzentwicklung und Ausbildung

Die Berufsausbildung und das Studium in allen Fachrichtungen dieser Berufe verschafft eine solide Basis zum Erhalt der hervorragenden Qualität des deutschen Geoinformationswesens. Dem bereits jetzt spürbaren Fachkräftemangel muss durch eine Ausbildungskampagne entgegengewirkt werden.

- Geoinformation zur Förderung der Wirtschaft

Die Wirtschaft setzt in vielen Prozessen auf Geoinformationen, wo sie mittlerweile unverzichtbar geworden sind. Die Märkte für Dienste und Produkte aus Geodaten und Satellitendaten gewinnen weiterhin an wirtschaftlicher Bedeutung und sind Vorreiter in den Technologiebereichen der Digitalisierung und der OpenData-Strategien.

- Geoinformationstechnologien zum Ausbau von Monitoringdiensten

Das volle Potenzial der europäischen Copernicus-Daten und moderner Geoinformationstechnologien ist für Akteure in Deutschland bekannt und nutzbar zu machen.

Eine vollständige Fassung des Programms lässt sich auch auf www.programm.ddgi.de oder unter dem angegeben QR-Code finden.

(aus Presse und DDGI-Auftritt) Zusammenarbeit in Europa nutzen

Europa ist bei der INSPIRERichtlinie an einem wichtigen Punkt der Umsetzung angelangt. So kann der europäische Gedanke gefestigt und zum nachhaltigen Aufbau von harmonischen Strukturen genutzt werden.

\section{Persönliches \\ Günter Schlemminger
1932-2018}

Günter Schlemminger, geachtetes und mit über 60 Jahren langjähriges Mitglied der DGfK ist am 16. Januar 2018 verstorben.

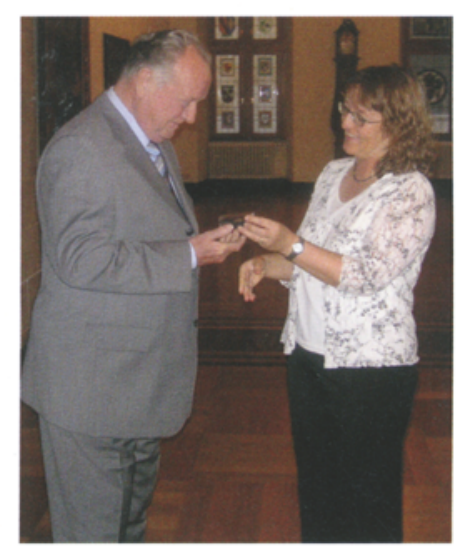

Sektionsleiterin Bettina Schmücking überreicht Günter Schlemminger die Medaille für 50 Jahre Mitgliedschaft in der DGfK (Foto: Walter Stupp)

Günter Schlemminger wurde am 24.5.1932 in Eydtkuhnen im nordöstlichen Ostpreußen geboren. Nach einer dramatischen Flucht im Herbst 1944 über das Haff und die Kurische Nehrung gelangte er nach Cossow in Mecklenburg. 1950 siedelte er nach Berlin-West über, wo er das Abitur ablegte und anschließend ein Ingenieurstudium absolvierte. 1957 wurde er durch Erlass des Senators für Wissenschaft und Forschung Berlin diplomiert.

Erste Berufserfahrungen machte Günter Schlemminger als Entwurfskartograph vom Mai 1957 bis Juli 1958 beim Verlag Herder Freiburg im Lexikographischen Institut, Abteilung Physische Kartographie. Nach Fertigstellung des „Großen Herder Atlasses“ war Günter Schlemminger dann ab September 1958 bis 1966 im Kartographischen Institut Bertelsmann in Gütersloh tätig.

Ende 1965 kam Günter Schlemminger wieder zurück nach Frei- 